\title{
Integrated Aperture Array Antenna Design for Radio Astronomy
}

\author{
Y. Zhang and A. K. Brown
}

\begin{abstract}
Microwave and Communication Systems Group, School of Electrical and Electronic Engineering, The University of Manchester, Manchester, M60 1QD, U.K. e-mail: david.zhang@ieee.org, anthony.brown@manchester.ac.uk *

Abstract. This paper describes the design of high performance compact aperture array antennas. A variety of existing microwave antenna designs has been investigated. The new microwave antenna designs suitable for SKA mid-frequency dense aperture array scenario are reported and their performances are compared to a new converted Vivaldi based array. Two new antenna element types including Bunny Ear Combline Antenna and Octagon Rings Antenna are proposed. The new designs use two different geometries-the BECA array is a 3-D structure whereas the ORA array forms a planar structure. All designs are analysed with a direct mounted front end. Performances for the new designs are compared. Guidelines are given for large scale wide band dual-polarized array designs in applications where a low cross polarization and a wide range of scan are required.
\end{abstract}

\section{Introduction}

Wide band dual-polarized aperture arrays are increasingly requested for various applications. Many system descriptions have well defined requirements for range of scans and polarization performance. Generally, wide range of scans and low cross polarization are desired across the wide operational bandwidth.

The element separation in terms of wavelength is one of the key parameters which has a significant impact on the array performance. It is directly related to mutual coupling between the neighbouring elements. For the conventional Vivaldi Tapered Slot Antenna (TSA), the design seeks to minimise the coupling to avoid impedance anomalies for large scan angles. In recent development of the wideband array design, mutual coupling is intentionally utilised and controlled between the array elements. The impedance stability over the frequency band and scan angles can be obtained by properly managing the capacitive coupling between the elements.

The stripline-fed Vivaldi TSA was widely used for its wideband wide-scan capability. However, the stripline-fed TSA produces high cross polarization components in the $45^{\circ}$ diagonal cut. A "bunny-ear" design has been reported in Lee et al. (2003) with its low- $Q$ structure for a better cross polarization performance. It is indicated in Lee et al. (2003) that this high cross-polarization components mainly stem from extensive surface current flowing in the longitudinal direction along the conductive tapered slot, and partly from unbalanced feed ports. A low profile Bunny Ear Combline Antenna (BECA) is proposed to improve the cross polarization performance over the entire scan range.

Elements which present a vertical conductor to the incoming field, e.g. the Vivaldis or other similar tapered slot structures, often suffer from high cross polarization in the $D$-plane, especially for the taller structures where a broader bandwidth is desired. Munk has shown that an array of dipoles closed to a ground plane and linked by capacitors can have a very wide-

\footnotetext{
* This work was supported by the European Commission Framework Program 6, Project SKADS, Square Kilometre Array Design Studies (SKADS), contract no 011938.
}

band active impedance (Munk 2006). The Munk dipole array is a planar structure. This is significantly different to a 3-D geometry for TSA arrays. A lower cross polarization over a wide usable frequency bandwidth are desired in the scan range from this new structure.

Mutual coupling always exists between the neighbouring elements and it is directly related to the element type, the element spacing in terms of wavelength and the array geometry. In the Vivaldi arrays, the element spacing may be less than one-tenth of the free-space in the case of 5:1 bandwidth array at the low frequency end (Chio \& Schaubert 2000). Mutual coupling can be large and this may cause impedance anomalies within the operational bandwidth and scan volume. As a result, the design seeks to quantify the coupling. The corrugated comblines between the tapered slots are then introduced to control the coupling by tuning the geometry and number of the comblines.

Munk antennas use a fundamentally different approach to design the wideband array (Munk 2003). Mutual coupling is intentionally utilized between the array elements, and controlled by the introduction of capacitance. Initially, work starts with coupled dipoles. The capacitance between the ends of dipoles make the field continuous and therefore a broad bandwidth can be obtained. The impedance stability over the frequency band and wide scan angles is enhanced by placing dielectric layers above the dipole array.

The superimposed dielectric layers are important to the design of the Munk dipole array. Three or four layers of dielectric slabs are compulsory in order to achieve a broad bandwidth. Cost becomes high for a large scale array in practice. Hence, elements with non-dipole shape are employed, preferably, the elements are octagonal rings in pairs. Furthermore, a meta material layer forming the same pattern as the array elements has been placed above the array to replace the layers of dielectric slabs. The array height can be significantly reduced.

This paper is organized as follows: In Section II, The tapered slot antenna and the octagonal ring antenna are introduced. Section III describes the fabricated finite array configurations for verification. The cross polarization performance is 
investigated in Section IV while Section V gives the information about the immersed element pattern and the scanned array pattern of the finite arrays. Section VI concludes the paper.

\section{Aperture array antenna elements}

\subsection{Design of Tapered Slot Antennas}

It is known that the conventional tapered slot Vivaldi antennas produce high cross polarization components in the diagonal $(D$ )plane. The antenna element with a "bunny ear" shape transformed from the conventional tapered slot antenna has been proposed for its low profile. The bunny ear antenna can produce a better cross polarization performance by tapering the outer edge of the conducting plate at the back end, and as a result, the element depth can be significantly shorter compared to a conventional Vivaldi antennas. The shorter element depth of the bunny ear design reduces the possibility of producing complex propagation modes along the conducting plates. In addition, the conducting flares of the bunny ear antennas are corrugated along the outer edge forming a combline shape. Such an antenna is named as Bunny Ear Combline Antenna (BECA). The outline profile for the proposed BECA antenna is shown in Fig. 1. The fabricated $16 \times 16$ dual polarized BECA array is shown in Fig. 2.

\subsection{Octagon Rings Antenna}

It is reported that an array of dipoles close to a groundplane can achieve a bandwidth of around 4:1 with a VSWR of less than 2:1 (Munk et al. 2003). However, the inter-element spacing should be small, smaller than a half wavelength at the high frequency. For applications where minimum number of elements are required, e.g. Square Kilometre Array (SKA), the largest allowable spacing(the element spacing avoiding grating lobes at maximum scan angle at the highest frequency) between the elements is desired. The bandwidth is reduced due to the limitations of wide-angle scans when the largest spacing is used. A new antenna structure is invented by placing a plurality of polygonal facets in a specified pattern. Metal patches with predetermined shapes locate on the top of the array with a relative distance to keep scan stability. The element facets and the metal patches on the top of array can be any polygonal shapes however the octagon rings preferably demonstrate an optimised performance. This particular design is named as "Octagon Rings Antenna (ORA)" in this paper. The fabricated mini size $4 \times 4$ ORA array is shown in Fig. 3 and the agreement between the simulated and experimental results is good. For an infinite ORA array with $165 \mathrm{~mm}$ element separation, the scan performance is shown in Fig. 4. Two approaches to implement the capacitive coupling between the element rings in the array are compared. Fig. 4a shows the performance of the array by using a $1 \mathrm{pF}$ bulk capacitor soldered between the elements, where $L_{g}$ is the distance between the element rings and the groundplane, $h$ is the distance from the element rings to the meta material layer and $s f$ is the ratio between the size of a meta material ring and an element ring. The interdigitated fingers are used for the array performance shown in Fig. 4b.

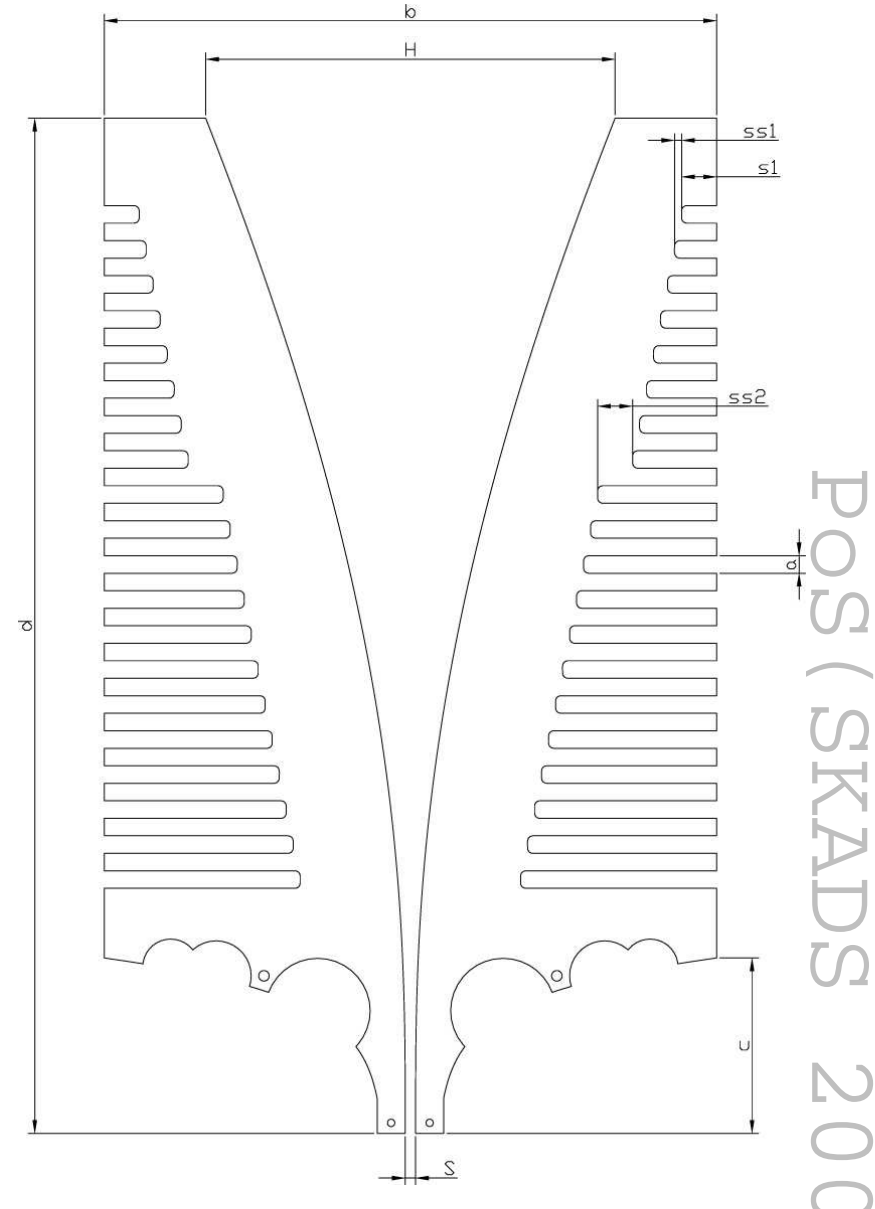

Fig. 1: The outline profile of a BECA antenna.

A fabricated $16 \times 16$ ORA array is shown in Fig. 5 and it has been measured in the Compact Antenna Test Range (CATR) at SELEX Galileo together with the BECA finite array.

\section{Finite elements array analysis}

In practice, such antenna arrays are not infinite in size. In order to study the performance of a single element in an infinite array environment, the number of the neighbouring elements needs to be large. Preliminary investigations indicate that the size of the array aperture is required to be greater than two and a half wavelength at the lowest frequency to reduce the edge effect to an acceptable level. A $16 \times 16 \times 2$ dual polarized BECA array was built and this has been shown in Fig. 2. The element separation is $175 \mathrm{~mm}$ in the array and therefore the aperture of the array is $2800 \mathrm{~mm}$. The height of the BECA element of $280 \mathrm{~mm}$ is less than one wavelength at the highest frequency and this is not typical for tapered slot type of antennas. A transformed design of differentially fed Vivaldi with the element height of $355 \mathrm{~mm}$ has been launched by ASTRON. The tapered slotline of the Vivaldi antenna has been fed directly without the coupling mechanism between the stripline and the slotline. A $16 \times 16 \times 2$ finite array has been built with the element separation of $160 \mathrm{~mm}$ and the array aperture of $2560 \mathrm{~mm}$. 


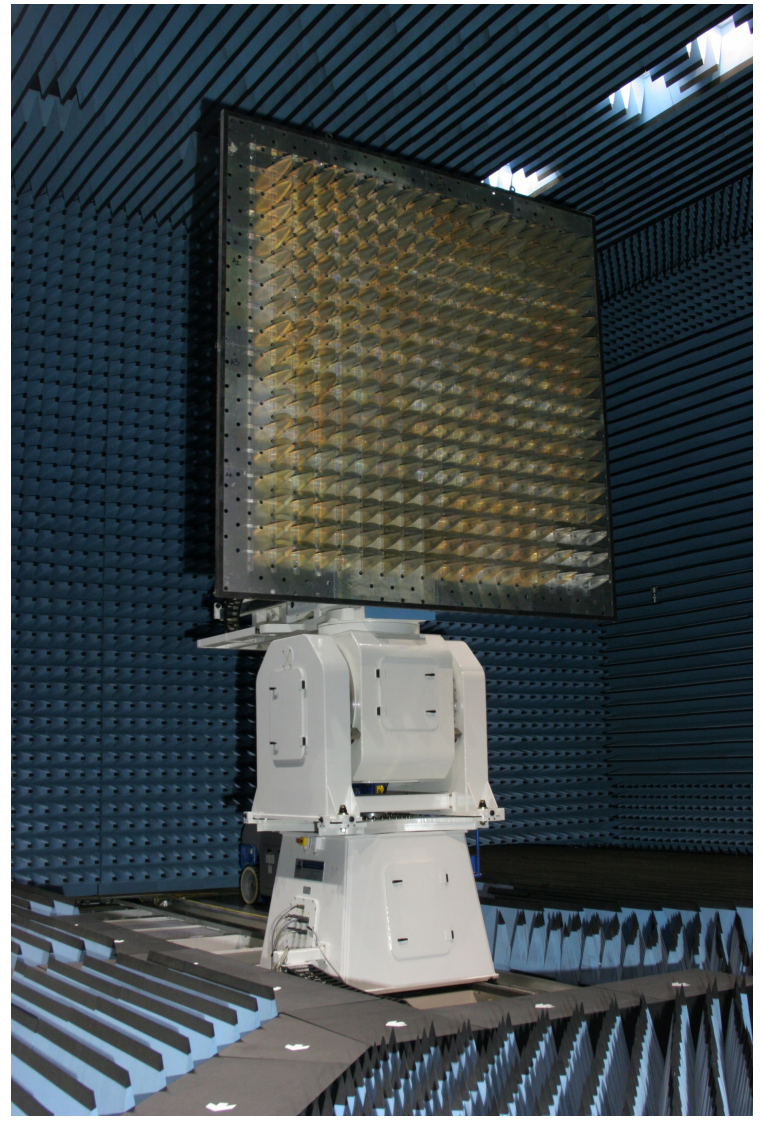

Fig. 2: The $16 \times 16$ BECA array, photo courtesy SELEX Galileo

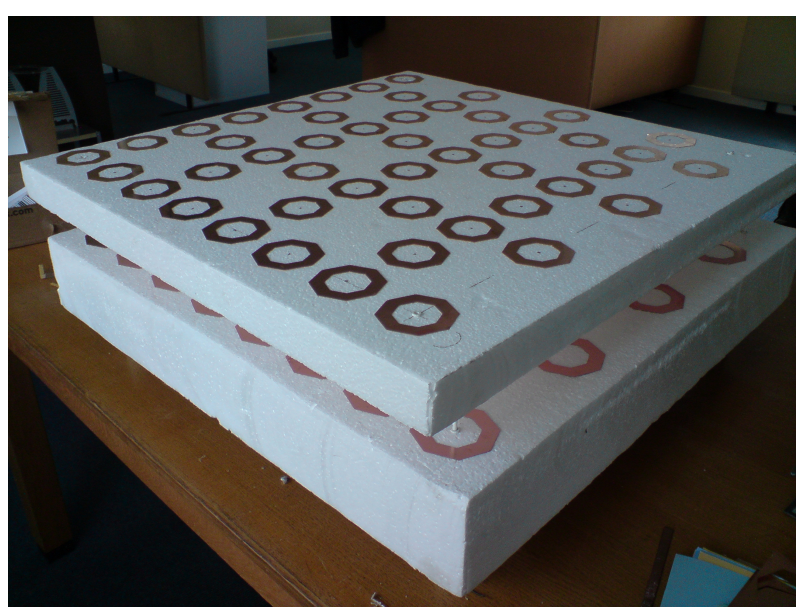

Fig. 3: The $4 \times 4$ finite ORA array.

In addition, a $16 \times 16 \times 2$ finite ORA was built and it is shown in Fig. 5. The element spacing is $165 \mathrm{~mm}$ and the capacitance value for the bulk capacitors between the elements is $1 \mathrm{pF}$. Three arrays have been measured in the compact antenna test range at SELEX Galileo following the same criteria. The active element pattern and scanned array patterns are investigated in the following sections.

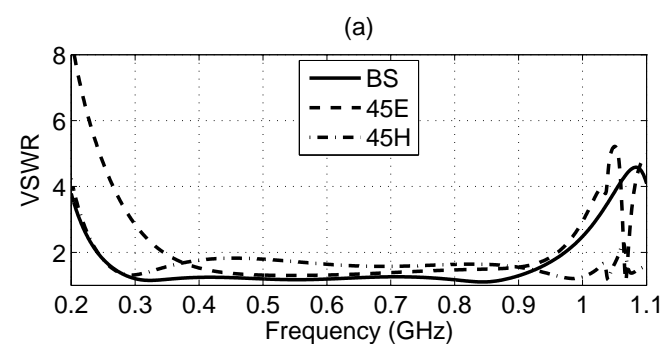

(b)

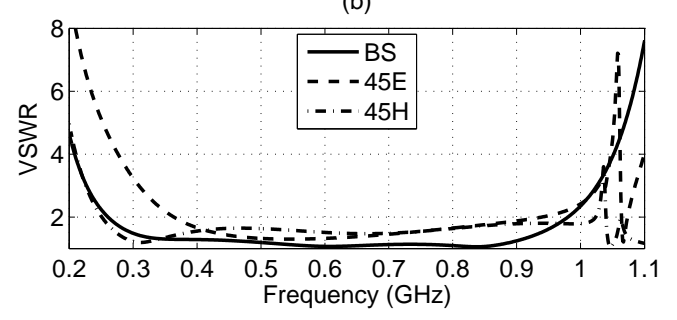

Fig. 4: The ORA with $1 \mathrm{pF}$ capacitor or interdigitated capacitors of 12 fingers, $h=70 \mathrm{~mm}, L_{g}=110 \mathrm{~mm}, \mathrm{sf}=0.9$ (a) $1 \mathrm{pF}$ capacitor; (b) Interdigitated capacitor with 12 fingers.

\section{Cross Polarization}

The Ludwig third definition of cross polarization is used in this paper (Ludwig 1973). The measured radiation patterns in the $45^{\circ}$ diagonal cut for the centre element of BECA and ORA finite arrays are shown in Fig. 6 and Fig. 7 respectively. In general, a longer tapered slot for the Vivaldis is required to achieve a broader bandwidth. However, the cross polarization performance degrades as the tapered slot becomes longer. The cross polarization for BECA antenna arrays with different element heights are compared with a conventional Vivaldi antenna array having the same element spacing. The cross polarization performances are shown in Fig. 8. The Vivaldi antenna element used here is a triplate structure. It indicates that an element with a shorter tapered slot produces a lower cross polarization, especially significant in the high frequency band. The cross polarization in the $D$-plane scan at three typical frequencies for an infinite ORA array is shown in Fig. 9. The comparison shows that ORA produces a lower cross polarization than that of the BECA in the $D$-plane. The ORA array shows a low and smooth cross polarization performance over the entire scan range. It is noted that the array exhibits the best cross polarization at the centre of the frequency band.

\section{Element and Array Pattern}

The active element pattern can be used to predict the performance of large phased array antennas and prevent array design failure before the large array system is fabricated. The active element pattern is taken when a single element in the array (the centre element) is fed, and the rest of elements are terminated in matched loads (Pozar 1994). The active element pattern is in general different from the radiation pattern for an isolated element. This is due to the mutual coupling effect between the neighbouring elements and the fed element and adjacent ele- 


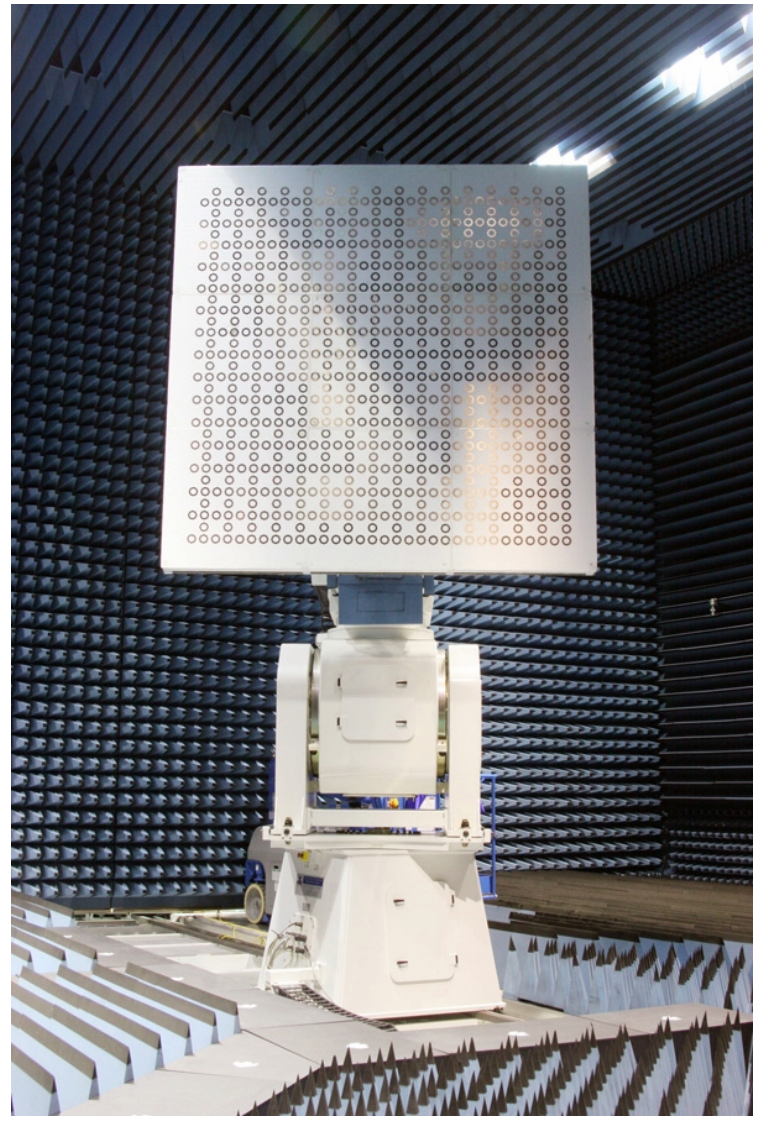

Fig. 5: The $16 \times 16$ finite ORA array, photo courtesy SELEX Galileo.

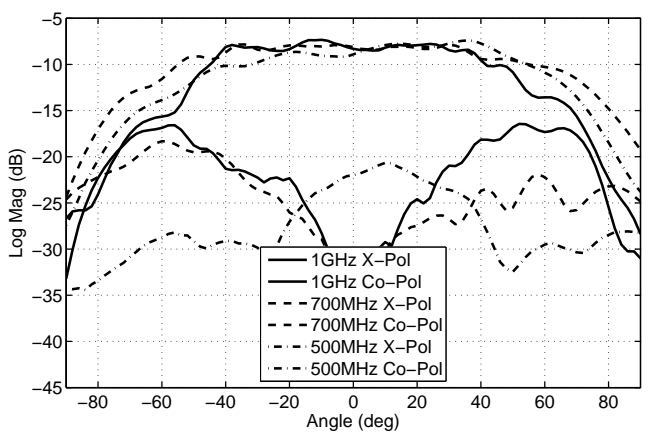

Fig. 6: The cross polarization in the D-plane, finite BECA array.

ments will radiate some power although they are not fed. The active element pattern can be assumed to be the same for each element in the array if the built array is large enough. The pattern of the phased array is the product of the active element pattern and the array factor.

The active element pattern for the centre element of finite BECA array is shown in Fig. 10. For the $16 \times 16 \times 2$ finite dual polarized ORA array, the active element pattern for the centre element at three frequencies is shown in Fig. 11. These measurements have been conducted in the presence of CPS-CPW baluns and their effects are included, a balanced signal was as-

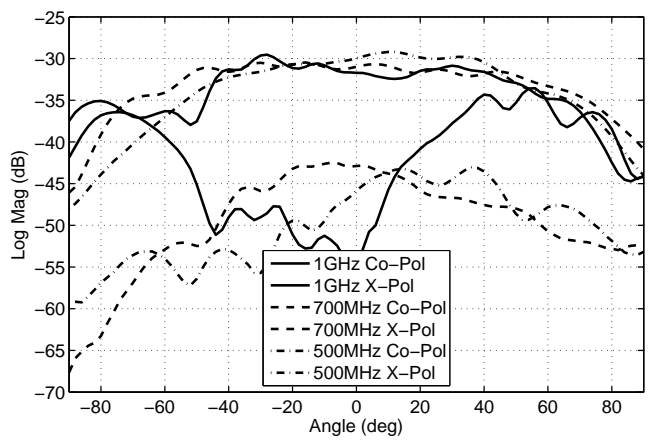

Fig. 7: The cross polarization in the D-plane, finite ORA array.

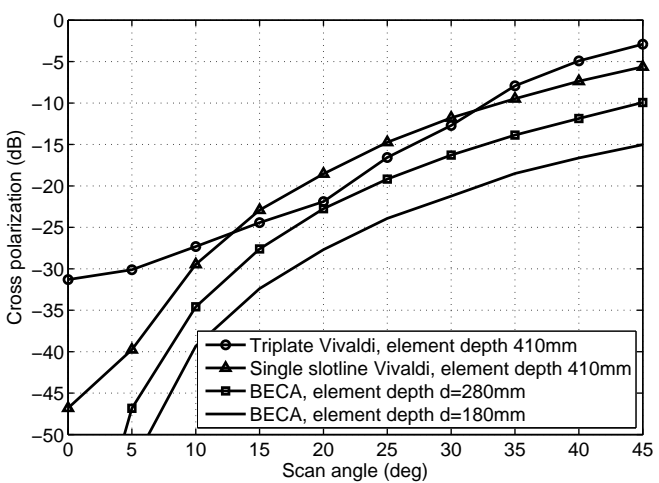

Fig. 8: The cross polarization in the D-plane at $1 \mathrm{GHz}$, infinite array.

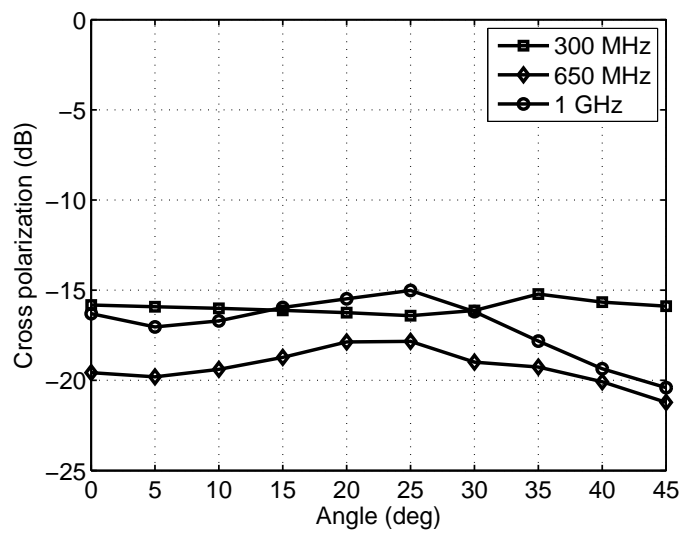

Fig. 9: The cross polarization in the D-plane, infinite ORA array, $150 \mathrm{~mm}$ element spacing.

sumed at the feed terminals on the balanced antennas. In addition to the active element pattern measurement, the subarray with $8 \times 8$ elements becomes active and the scanned array pattern is shown in Fig. 12. The loss of gain with scan from broadside to $45^{\circ}$ in the principle planes is less than $1.5 \mathrm{~dB}$ with a slightly more scan loss in the $E$-plane than in the $H$-plane. The ORA is shown to have less loss of gain with scan in the $\pm 45^{\circ}$ scan range. 


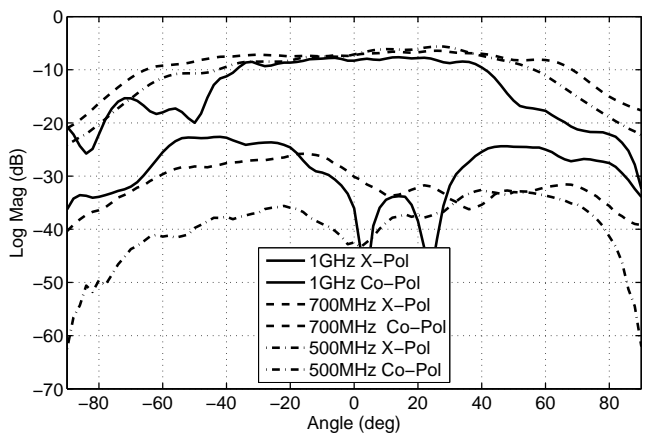

(a)

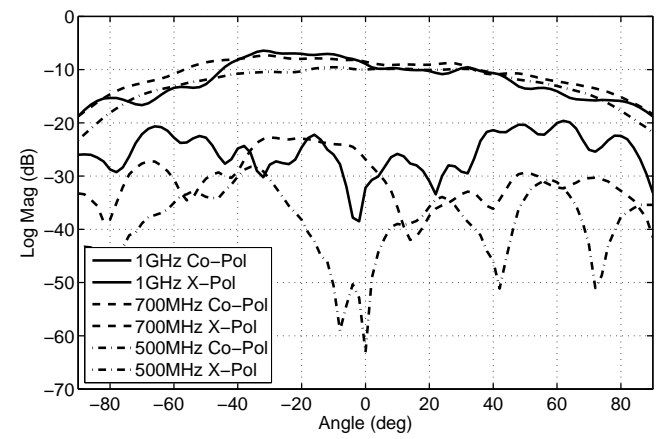

(b)

Fig. 10: The immersed centre element pattern of the $16 \times 16$ finite BECA array (a) $E$-plane, (b) $H$-plane.

\section{Conclusions}

The paper investigates the radiating element design for wideband aperture arrays. The arrays studied consist of the main conventional tapered slotline Vivaldi antenna, the proposed bunny-ear combline antenna and the latest developed octagon ring antenna with a planar structure. The ORA shows an overall better performance including broader element patterns and lower cross polarization in addition to a lower cost and easy implementation. The array exhibits $4: 1$ bandwidth and $-15 \mathrm{~dB}$ cross polarization within a $\pm 45^{\circ}$ scan volume. These elements are initially designed for the radio astronomy application operating from $300 \mathrm{MHz}$ to $1000 \mathrm{MHz}$. However, they are readily scalable to other higher frequency range for various applications.

Acknowledgements. The authors would like to give special thanks to Dr. Georgina Harris, Tim Ikin and Chris Shenton for their efforts and support on fabrication and measurement of the finite arrays.

\section{References}

Chio, T-H. \& Schaubert, D. H. 2000, IEEE Trans. Antenna Propagat., 48, 879

Lewis, L. R., Fasset, M. \& Hunt, J. 1974, IEEE Int. Symp. Antennas Propagat., 335

Lee, J. J., Livingston, S. \& Koenig, R. 2003, IEEE Antennas And Wireless Propagat. Letters, 2, 46

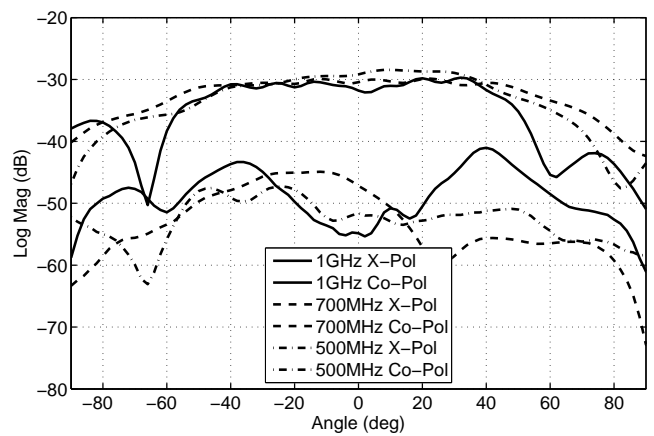

(a)

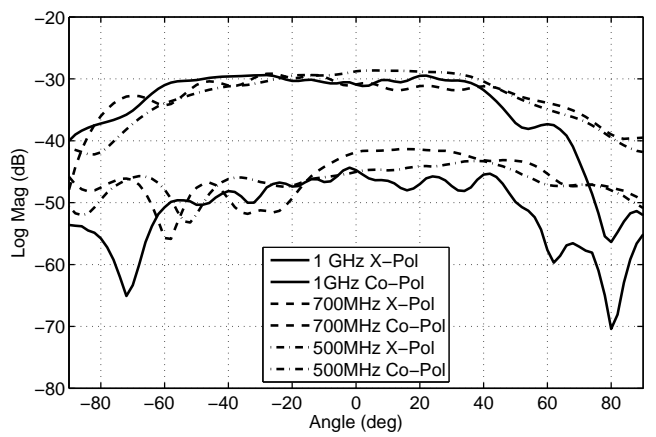

(b)

Fig. 11: The immersed centre element pattern of the $16 \times 16$ finite ORA array (a) $E$-plane, (b) $H$-plane.

Ludwig, A. C. 1973, IEEE Trans. Antenna Propagat., 116 Munk, B. A. 2003, Finite Antenna Arrays and FSS

Munk, B. A., Taylor, R., Durham, T., Croswell, W., Pigon,

B., Boozer, R., Brown, S., Jones, M., Pryor, J., Ortiz,

S., Rawnick, J., Krebs, K., Vanstrum, M., Gothard, G., \& Wiebelt, D. 2003, In Proceedings of Antennas and

Propagation Society International Symp. 2003, 448

Munk, B. A. 2006, EuCAP 2006, 149

Pozar, D. M. 1994, IEEE Trans. Antenna Propagat., 42, 1176 


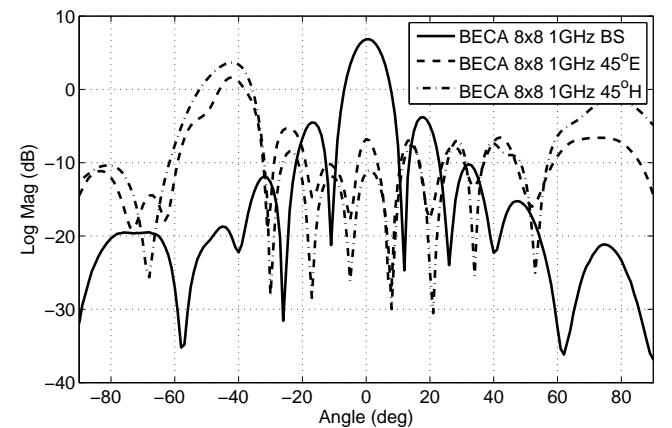

(a)

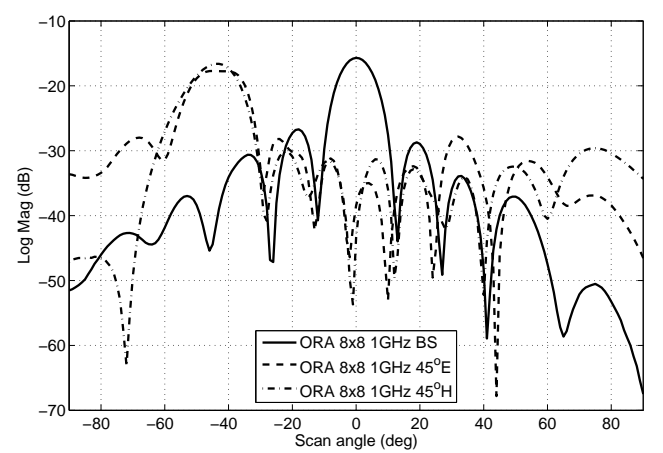

(b)

Fig. 12: The scanned array pattern for the $8 \times 8$ subarray, (a) BECA, (b) ORA. 